\title{
Correlation of the controlling nutritional status score and the prognostic nutritional index with the prognosis of patients treated with radiotherapy for small-cell lung cancer
}

\author{
Lina $\mathrm{Li}^{1 \#}$, Yeling Wang ${ }^{2 \#}$, Ping Yang ${ }^{3}, \mathrm{Lu} \mathrm{Xu}^{1}$, Shufang Liu ${ }^{1}$, Shuang Zhang ${ }^{2}$, Xiangqian Weng $^{2}$ \\ ${ }^{1}$ Department of Hematology, the First Affiliated Hospital of Hainan Medical University, Haikou, China; ${ }^{2}$ Department of Radiotherapy, the First \\ Affiliated Hospital of Hainan Medical University, Haikou, China; ${ }^{3}$ Radiotherapy Ward, the First Affiliated Hospital of Hainan Medical University, \\ Haikou, China \\ Contributions: (I) Conception and design: L Li, Y Wang, X Weng; (II) Administrative support: P Yang, L Xu; (III) Provision of study materials or \\ patients: L Li, Y Wang, X Weng; (IV) Collection and assembly of data: L Li, Y Wang, X Weng; (V) Data analysis and interpretation: L Li, X Weng; (VI) \\ Manuscript writing: All authors; (VII) Final approval of manuscript: All authors. \\ "These authors contributed equally to this work. \\ Correspondence to: Xiangqian Weng. Department of Radiotherapy, the First Affiliated Hospital of Hainan Medical University, 31 Longhua Road, \\ Haikou 570102, China. Email: wengxiangqian5151@163.com.
}

Backgroundk We aimed to explore the associations between the controlling nutritional status (CONUT), the prognostic nutritional index (PNI), and prognostic outcomes in patients with small-cell lung cancer (SCLC) receiving radiotherapy.

Methods: A total of 93 SCLC patients who received radiotherapy at the hematology department in the First Affiliated Hospital of Hainan Medical University were retrospectively included in this analysis. Patient CONUT and PNI values were calculated. Receiver operating characteristic (ROC) curves were utilized to determine the optimal cut-off, the area under the curve (AUC), sensitivity, and specificity of CONUT and PNI in discriminating patient outcomes. Kaplan-Meier curve analysis was performed, and overall survival (OS) was compared for patients with CONUT $>3$ and $\leq 3$, or those with PNI $>47.7$ and $\leq 47.7$. Univariate and multivariate analysis using the Cox proportional hazard model was performed to identify the risk factors for patient death.

Results: The AUCs for CONUT and PNI were 0.95 and 0.82 , respectively. The CONUT results indicated that patient OS decreased significantly from normal to mildly malnourished. Meanwhile, the OS for moderately malnourished patient decreased to severely malnourished ( $\mathrm{P}$ for trend $<0.001$ ). Patients with CONUT $>3$ or PNI $\leq 47.7$ had significantly shorter OS than those with CONUT $\leq 3$ (median survival time 12 vs. 23 months; $\mathrm{P}<0.001$ ) or with $\mathrm{PNI}>47.7$ (median survival time 20 vs. 15 months; $\mathrm{P}=0.010$ ). Multivariate analysis indicated that the TNM stage [hazard ratio (HR) $=1.21 ; 95 \%$ confidence interval (CI): 1.03-1.66], CONUT score (HR $=2.33$; 95\% CI: 1.76-3.91), and PNI $\leq 47.7$ (HR $=1.33$; 95\% CI: 1.09-1.99) were independent prognostic indicators for patient death.

Conclusions: The CONUT and PNI were potential indicators for the outcome of patients with SCLC. CONUT $>3$ or PNI $\leq 47.7$ indicated an unfavorable prognosis for SCLC patients.

Keywords: Controlling nutritional status (CONUT); prognostic nutritional index (PNI); small-cell lung cancer (SCLC); radiotherapy

Submitted Aug 31, 2021. Accepted for publication Oct 29, 2021.

doi: 10.21037/apm-21-2740

View this article at: https://dx.doi.org/10.21037/apm-21-2740 


\section{Introduction}

Small-cell lung cancer (SCLC) is a common pathological subtype of lung cancer, often found in elderly patients. It is characterized by sensitivity to chemotherapy/radiotherapy, rapid tumor growth, early metastasis, and poor prognosis. At present, an increasing number of studies have shown that the nutritional status and immune function of tumor patients have a close relationship with the prognostic outcome of patients (1). In addition, SCLC radiotherapy can easily cause discomforts such as anorexia and loss of appetite, which can lead to a series of problems such as malnutrition. The controlling nutritional status (CONUT) score is a brief index proposed in 2005 to assess the nutritional status of patients and includes three indices: serum albumin, lymphocyte count, and cholesterol. The prognostic nutritional index (PNI) consists of two indices, namely serum albumin and lymphocyte count. Previous literature has confirmed that CONUT and PNI can reflect the nutritional and immune status of patients, and can assist in evaluating the risk of surgery as well as the risk of postoperative complications in patients with many types of tumors $(2,3)$. Recent studies have shown that CONUT and PNI can serve as potential indicators for predicting the prognosis of SCLC $(4,5)$. At present, However, the current research on the use of CONUT and PNI to assess the prognosis of SCLC patients with radiotherapy focuses on a single indicator, and rarely involves the simultaneous evaluation of multiple nutritional indicators. This study aimed to investigate the role of these two nutrition immune indicators in the prognostic evaluation of patients treated with radiotherapy for SCLC. We present the following article in accordance with the STARD reporting checklist (available at https://dx.doi.org/10.21037/apm-21-2740).

\section{Methods}

\section{Subjects}

A total of 93 patients with SCLC who underwent radiotherapy at the hematology department in the First Affiliated Hospital of Hainan Medical University from January 2018 to December 2019 were included in this retrospective study. Inclusion criteria: (I) patients with SCLC diagnosed pathologically; (II) patients had routine blood and biochemical test results within 1 month before radiotherapy; (III) patient clinical data was complete. Exclusion criteria: (I) patient life expectancy <4 weeks; (II) patients had combined secondary tumors of other systems; (III) patients with comorbidities which might affect lymphocyte counts in hematological disorders, such as aplastic hematological disorders. All procedures performed in this study involving human participants were in accordance with the Declaration of Helsinki (as revised in 2013). The study was approved by the Ethics Committee of the First Affiliated Hospital of Hainan Medical University (No. 2017O612) and informed consent was obtained from all the patients.

\section{Clinical data collection and follow up}

The basic information and laboratory findings of patients, including age, sex, smoking history, TNM stage, and Eastern Cooperative Oncology Group (ECOG) performance score, were collected by our hospital's electronic medical record system. All patients were followed until death or loss to follow-up, and telephone follow-up was set for March 2021. Patient overall survival (OS) time was defined as the time from receiving radiotherapy to the date of death or follow-up cut-off.

\section{CONUT and PNI computational methods}

The CONUT score was determined by adding up the three indexes of serum albumin, lymphocyte count, and cholesterol level individually. Specifically, albumin $\geq 3.5$, $3.0-3.49,2.5-2.99$, and $<2.5 \mathrm{~g} / \mathrm{dL}$ were each counted on a scale of $0,2,4$, and 6 points, respectively. Lymphocyte counts $\geq 1,600,1,200-1,599,800-1,199$, and $<800$ cells $/ \mathrm{mL}$ were counted for $0,1,2$, and 3 points, respectively. Total cholesterol >180, 140-180, 100-139, and <100 mg/dL were similarly counted as $0,1,2$, and 3 points, respectively. It is generally accepted that a score of $0-1$ indicates normal nutrition, while scores of $2-4,5-8$, and $\geq 9$ indicate mild, moderate, and severe malnutrition, respectively. PNI was counted as PNI $($ score $)=5 \times$ lymphocyte counts $\left(\times 10^{9} / \mathrm{L}\right)+$ serum albumin (g/L). PNI $\geq 50,45 \leq \mathrm{PNI}<50,40 \leq \mathrm{PNI}<45$, and PNI $<40$ were suggestive of normal, mild, moderate, and severe malnutrition, respectively.

\section{Statistical analysis}

Categorical data were presented as numbers or percentages. Normally distributed measures were expressed as mean \pm $\mathrm{SD}$ and non-normally distributed measures were expressed as median (quartile). With patient death as the outcome, the area under the curve (AUC), optimal cut-off value, 
Table 1 Demographics and general data of the included patients

\begin{tabular}{lc}
\hline Index & Value \\
\hline Age (years), mean \pm SD & $65.44 \pm 9.82$ \\
Sex (male/female) & $51 / 42$ \\
Smoking history, n (\%) & \\
Never smoker & $28(30.11)$ \\
Previous smoker & $35(37.63)$ \\
Current smoker & $30(32.26)$ \\
TNM stage, n (\%) & \\
I + II & $24(25.81)$ \\
III + IV & $69(74.19)$ \\
ECOG score, median [interquartile range] & $0[0-0.5]$ \\
NSE (ng/mL), mean \pm SD & $48.68 \pm 6.98$ \\
CONUT score, n (\%) & \\
$0-1$ & $18(19.35)$ \\
$2-4$ & $28(30.11)$ \\
$5-8$ & $30(32.26)$ \\
$\geq 9$ & $17(18.28)$ \\
PNI score, n (\%) & \\
250 & $42(45.16)$ \\
$40 \leq$ PNI $<50$ & \\
\hline & \\
\hline &
\end{tabular}

ECOG, Eastern Cooperative Oncology Group; NSE, neuronspecific enolase; CONUT, controlling nutritional status; PNI, prognostic nutritional index.

sensitivity, and specificity for PNI and CONUT were determined by receiver operating characteristic (ROC) curves. The trend test $\mathrm{P}$ value ( $\mathrm{P}$ for trend) was calculated by dividing the patients into normal, mild, moderate, and severe malnutrition groups based on CONUT and PNI results. Survival curves were plotted using the Kaplan-Meier method, and the log-rank test compared the difference in patient survival between the two groups with or without statistical significance. Univariate and multivariate analyses of risk factors for patient mortality were performed using Cox proportional hazards models. All tests were two-sided, and the test level was set at $\alpha=0.05$.

\section{Results}

\section{Patient general information}

The 93 included patients (51 males and 42 females) had a mean age of 65.44 years, and $74.19 \%$ of the patients were already at TNM stage III or IV at the time of diagnosis. The percentages of patients with a CONUT score indicating the presence of mild, moderate, and severe malnutrition were $29.04 \%, 45.16 \%$, and $19.35 \%$, respectively. In contrast, those with a PNI score indicating the presence of mild, moderate, and severe malnutrition were $30.11 \%$, 32.26\%, and $18.28 \%$, respectively (Table 1 ).

\section{Efficacy of PNI and CONUT in discriminating patient outcomes}

As shown in Figure 1A, when the optimal cut-off value was taken to be 47.7, the AUC of PNI to identify whether a patient was alive or not was 0.82 [95\% confidence interval (CI): $0.73-0.89$ ], with a sensitivity and specificity of $71.3 \%$ (95\% CI: 60.0-80.8\%) and 84.6\% (95\% CI: 54.6-98.1\%), respectively. As shown in Figure $1 B$, when the optimal cut-off value was taken as 3, the AUC of CONUT for discriminating whether patients were alive was $0.95(95 \%$ CI: $0.88-0.98)$, with a sensitivity and specificity of $82.5 \%$ (95\% CI: $72.4-90.1 \%)$ and $92.3 \%$ (95\% CI: 64.0-99.8\%), respectively.

\section{Association of PNI and CONUT with patient outcomes}

As shown in Table 2, when the nutritional status of patients was divided into normal, mildly malnourished, moderately malnourished, and severely malnourished according to the PNI results, the change in the OS of SCLC patients in each group was not statistically significant (P for trend $=0.228$ ). In contrast, according to the CONUT results, the OS of patients in each group decreased stepwise ( $\mathrm{P}$ for trend $<0.001)$.

\section{Kaplan-Meier survival analysis of SCLC patients}

By the end of follow-up, a total of $80(86.02 \%)$ patients had died. The median survival for all patients was 16 months. After dividing the patients into PNI $>47.7$ and $\leq 47.7$ groups according to the ROC results, the survival analysis 
A

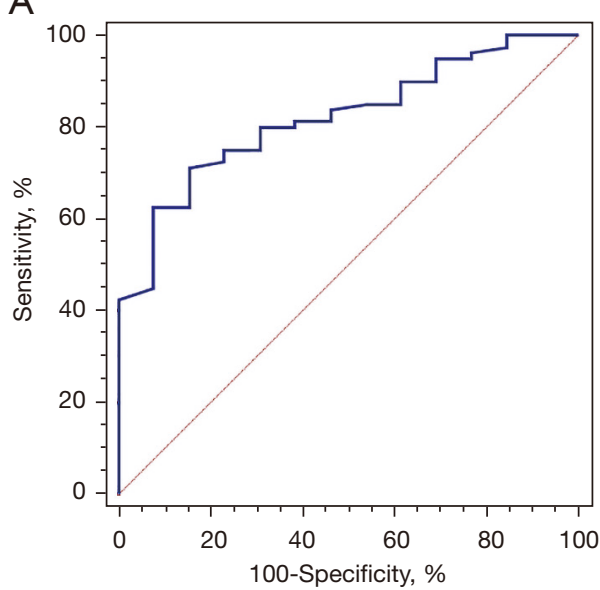

B

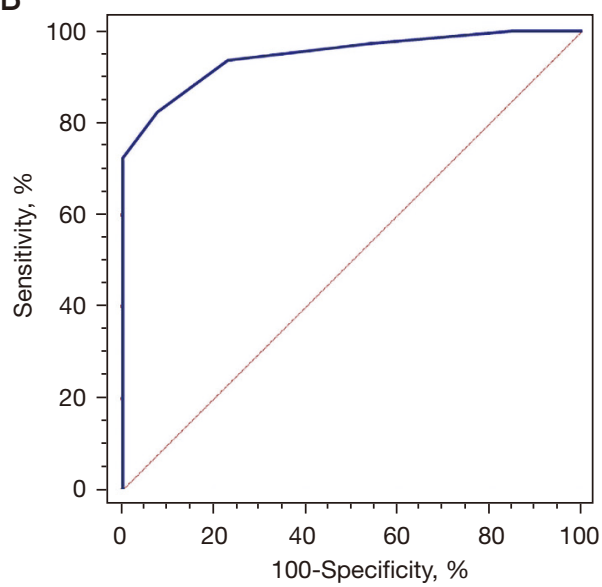

Figure 1 Evaluation of patient outcome with the ROC curve. The ROC curves for discriminating outcomes with the PNI (A) or CONUT score (B) in patients with SCLC undergoing radiotherapy. ROC, receiver operating characteristic; PNI, prognostic nutritional index; CONUT, controlling nutritional status; SCLC, small-cell lung cancer.

Table 2 Comparison of OS according to the nutritional status defined by the PNI and the CONUT score

\begin{tabular}{lcccc}
\hline Index & Normal & Mildly malnourished & Moderately malnourished & Severely malnourished \\
\hline PNI (month), mean \pm SD & $17.67 \pm 6.95$ & $15.46 \pm 8.23$ & $14.93 \pm 7.45$ & $12.00 \pm 7.61$ \\
CONUT (month), mean \pm SD & $23.88 \pm 3.52$ & $16.93 \pm 7.25$ & $12.43 \pm 7.24$ & $14.56 \pm 7.55$ \\
\hline
\end{tabular}

OS, overall survival; PNI, prognostic nutritional index; CONUT, controlling nutritional status.
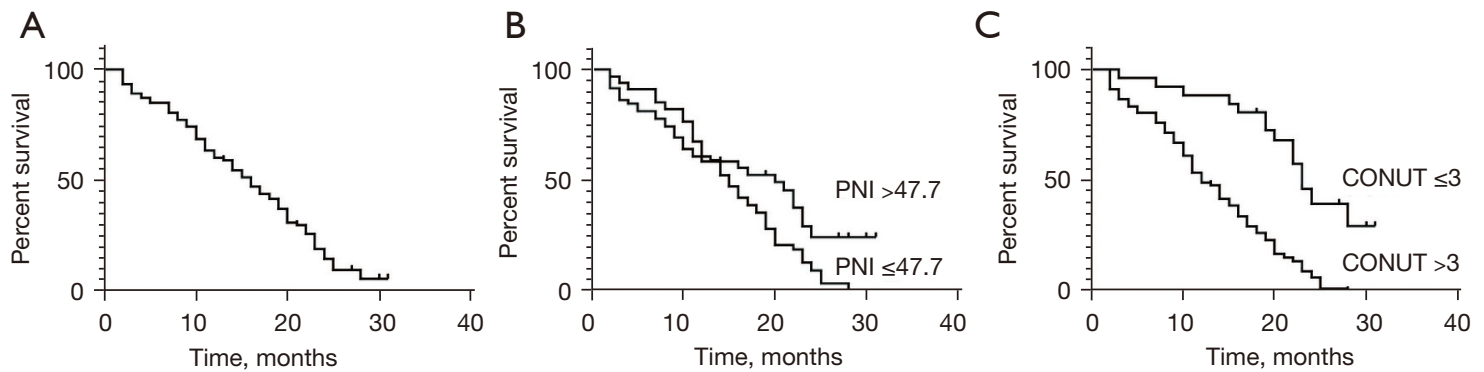

Figure 2 The Kaplan-Meier curve survival analysis of SCLC patients. (A) The Kaplan-Meier curve for the OS of SCLC patients undergoing radiotherapy. (B,C) Comparisons of the OS of SCLC patients undergoing radiotherapy according to the PNI (B) or the CONUT score (C). SCLC, small-cell lung cancer; OS, overall survival; PNI, prognostic nutritional index; CONUT, controlling nutritional status.

(Figure 2) suggested that the median survival of patients in the former group was significantly longer than that in the latter group (20 vs. 15 months; $\mathrm{P}=0.010)$. Similarly, when dividing patients into two groups with CONUT $>3$ and $\leq 3$, patients in the former group had a significantly shorter median survival than those in the latter group (12 vs. 23 months; $\mathrm{P}<0.001$ ).

\section{Univariate and multivariate analysis of death in SCLC patients}

As shown in Table 3, the univariate analysis suggested that higher TNM stage, ECOG score $>3$, CONUT score $>3$, and PNI $\leq 47.7$ were risk factors for mortality in radiotherapy-treated SCLC patients. Multivariate 
Table 3 Univariate and multivariate analysis for the identification of risk factors for patient outcomes in SCLC patients undergoing radiotherapy

\begin{tabular}{|c|c|c|c|c|}
\hline Variables & \multicolumn{2}{|c|}{ Univariate analysis } & \multicolumn{2}{|c|}{ Multivariate analysis } \\
\hline Age (>69 vs. $\leq 69$ years) & $0.89(0.51-1.43)$ & 0.555 & & \\
\hline Sex (male vs. female) & $1.03(0.71-1.55)$ & 0.812 & & \\
\hline Smoking history (no vs. yes) & $1.36(0.91-1.71)$ & 0.311 & & \\
\hline$I+I I$ & Reference & & & \\
\hline III + IV & $1.54(1.34-1.76)$ & 0.009 & $1.21(1.03-1.66)$ & 0.047 \\
\hline \multicolumn{5}{|l|}{ ECOG score } \\
\hline 0 & Reference & & & \\
\hline 3 & $1.82(1.56-3.97)$ & 0.032 & $1.18(0.78-1.33)$ & 0.069 \\
\hline CONUT ( $\leq 3$ vs. $>3$ ) & $2.99(1.89-4.87)$ & $<0.001$ & $2.33(1.76-3.91)$ & 0.008 \\
\hline PNI (>47.7 vs. $\leq 47.7)$ & $2.03(1.21-2.76)$ & 0.003 & $1.33(1.09-1.99)$ & 0.021 \\
\hline
\end{tabular}

SCLC, small-cell lung cancer; HR, hazard ratio; Cl, confidence interval; ECOG, Eastern Cooperative Oncology Group; CONUT, controlling nutritional status; PNI, prognostic nutritional index.

regression analysis suggested that TNM stage [hazard ratio $(\mathrm{HR})=1.21 ; 95 \%$ CI: 1.03-1.66], CONUT score $>3$ (HR $=2.33$; 95\% CI: $1.76-3.91)$, and $\mathrm{PNI} \leq 47.7$ (HR $=1.33 ; 95 \%$ CI: 1.09-1.99) were independent risk factors for patient mortality.

\section{Discussion}

Malnutrition is a prevalent clinical manifestation in patients with tumors. Studies have found that about $30-80 \%$ of patients with tumors present with varying degrees of malnutrition $(6,7)$. Recent studies have suggested that the causes of malnutrition in cancer patients are involved in dysbiosis and decreased eating, as evidenced by impaired nutrient metabolism, increased body energy expenditure at rest, and changes in endogenous cytokine levels (8). An increasing number of studies have found that malnutrition is an independent risk factor for poor prognosis in patients with various malignancies (9). Thus, nutritional assessment and treatment of patients with tumors have been gradually valued.

In the present study, we used two common methods of nutritional status assessment, namely CONUT and
PNI. The PNI contains two indicators of serum albumin and lymphocyte count. Serum albumin concentration has a negative relationship with nutritional deficiencies, and can therefore be used as a surrogate marker to assess the nutritional status of patients. Lymphocytes promote apoptosis and inhibit cell proliferation through cytotoxicity, and their quantification can reflect the body's immune status to some extent. CONUT adds the additional indicator of cholesterol levels based on PNI. In cancer patients, hypocholesterolemia is more common, and is associated with increased LDLR expression, LDL endocytosis, and increased levels of oxidative phosphorylation (10). In addition, tumor dyscrasia can also often lead to hypocholesterolemia in patients. It is well established that nutritional status as assessed by PNI and CONUT is significantly associated with the prognosis of patients with several malignancies, including breast (11), colorectal (12), lymphoma (13), esophageal (14), pancreatic (15), and cervical cancer (16).

The present study found that the proportion of SCLC patients with moderate or severe malnutrition was $64.51 \%$ and $50.54 \%$, respectively, according to the CONUT and PNI results, which was similar to previous reports in other 
types of malignancies (17). In addition, the OS of patients decreased stepwise as the nutritional status determined by the CONUT deteriorated. Similarly, survival analysis suggested that SCLC patients with a CONUT $>3$ or PNI $\leq 47.7$ had a significantly shorter OS than those in the CONUT $\leq 3$ or $\mathrm{PNI}>47.7$ group, suggesting that the worse the nutrition of the patients, the shorter their OS. The effect of nutritional status on the prognosis of lung cancer patients has been reported in previous literature. For example, Wang and colleagues found that low PNI before treatment was associated with shorter OS, diseasefree survival, progression-free survival, and recurrencefree survival in non-SCLC (NSCLC) patients and was associated with decreased OS in SCLC patients in a metaanalysis containing 17 NSCLC and 4 SCLC studies (18). Jin et al. found that patient age, tumor TNM stage, and PNI were independent prognostic factors for the OS of SCLC patients (19).

To the best of our knowledge, the importance of the CONUT score for the prognostic evaluation of SCLC has been reported less frequently in previous literature. Toyokawa et al.'s study (20) showed that a high CONUT score was associated with shorter disease-free survival in lung squamous cell carcinoma patients. Yilmaz et al. compared CONUT with PNI for the prognosis of SCLC patients and found that the former was an independent risk factor for patient outcome. At the same time, the latter was not statistically significant in multivariate analysis (5). The results of multivariate analysis in this study indicated that TNM stage, CONUT, and PNI were independent risk factors for SCLC patients treated with radiotherapy. In addition, the nutritional level of patients is also an influential factor in the prognosis of patients with NSCLC (21-23). Therefore, in clinical practice, medical staff should pay attention to assessing the nutritional status of SCLC patients and provide targeted interventions in order to improve the prognosis of patients.

The limitations of this study were that it was designed as a retrospective analysis and had a small sample size. Other indicators of inflammation and nutritional-immune status, such as C-reactive protein, weight change, and skin thickness, were not included in this study. In addition, this study focuses on SCLC patients. Whether CONUT score and PNI can be used for prognostic evaluation of NSCLC lung cancer patients remains to be explored. Finally, although CONUT score and PNI have been widely accepted as prognostic indicators of malignant tumors, their relationship with other traditional indicators and whether they can be used as independent prognostic indicators remains to be verified.

In conclusion, this study analyzed the relationship between nutritional status and prognosis in patients with SCLC who underwent radiotherapy and found that the CONUT score and PNI were independent risk factors for patient outcome. A CONUT $>3$ or a PNI $\leq 47.7$ suggests a poor prognosis for the patient. This study reminds healthcare workers in clinical practice that nutritional status assessment and corresponding treatment should be paid attention to in the hope of improving patient outcomes.

\section{Acknowledgments}

Funding: The project was supported by Hainan Province Clinical Medical Center.

\section{Footnote}

Reporting Checklist: The authors have completed the STARD reporting checklist. Available at https://dx.doi. org/10.21037/apm-21-2740

Data Sharing Statement: Available at https://dx.doi. org/10.21037/apm-21-2740

Conflicts of Interest: All authors have completed the ICMJE uniform disclosure form (available at https://dx.doi. org/10.21037/apm-21-2740). The authors have no conflicts of interest to declare.

Ethical Statement: The authors are accountable for all aspects of the work in ensuring that questions related to the accuracy or integrity of any part of the work are appropriately investigated and resolved. All procedures performed in this study involving human participants were in accordance with the Declaration of Helsinki (as revised in 2013). The study was approved by the Ethics Committee of the First Affiliated Hospital of Hainan Medical University (No. 2017O612) and informed consent was obtained from all the patients.

Open Access Statement: This is an Open Access article distributed in accordance with the Creative Commons Attribution-NonCommercial-NoDerivs 4.0 International License (CC BY-NC-ND 4.0), which permits the non- 
commercial replication and distribution of the article with the strict proviso that no changes or edits are made and the original work is properly cited (including links to both the formal publication through the relevant DOI and the license). See: https://creativecommons.org/licenses/by-nc-nd/4.0/.

\section{References}

1. Capra S, Ferguson M, Ried K. Cancer: impact of nutrition intervention outcome--nutrition issues for patients. Nutrition 2001;17:769-72.

2. Ahiko Y, Shida D, Horie T, et al. Controlling nutritional status (CONUT) score as a preoperative risk assessment index for older patients with colorectal cancer. BMC Cancer 2019; 19:946.

3. Lee SC, Lee JG, Lee SH, et al. Prediction of postoperative pulmonary complications using preoperative controlling nutritional status (CONUT) score in patients with resectable non-small cell lung cancer. Sci Rep 2020;10:12385.

4. Ozdemir Y, Topkan E, Mertsoylu H, et al. Low prognostic nutritional index predicts poor clinical outcomes in patients with stage IIIB non-small-cell lung carcinoma undergoing chemoradiotherapy. Cancer Manag Res 2020;12:1959-67.

5. Yilmaz A, Tekin SB, Bilici M, et al. The significance of controlling nutritional status (CONUT) score as a novel prognostic parameter in small cell lung cancer. Lung 2020;198:695-704.

6. Bozzetti F, Mariani L, Lo Vullo S, et al. The nutritional risk in oncology: a study of 1,453 cancer outpatients. Support Care Cancer 2012;20:1919-28.

7. Hébuterne X, Lemarié E, Michallet M, et al. Prevalence of malnutrition and current use of nutrition support in patients with cancer. JPEN J Parenter Enteral Nutr 2014;38:196-204.

8. Argilés JM, Busquets S, Stemmler B, et al. Cancer cachexia: understanding the molecular basis. Nat Rev Cancer 2014;14:754-62.

9. de Pinho NB, Martucci RB, Rodrigues VD, et al. High prevalence of malnutrition and nutrition impact symptoms in older patients with cancer: results of a Brazilian multicenter study. Cancer 2020;126:156-64.

10. Parsa N, Taravatmanesh S, Trevisan M. Is low cholesterol a risk factor for cancer mortality? Eur J Cancer Prev 2018;27:570-6.

11. Chen L, Bai P, Kong X, et al. Prognostic nutritional index (PNI) in patients with breast cancer treated with neoadjuvant chemotherapy as a useful prognostic indicator. Front Cell Dev Biol 2021;9:656741.

12. Takamizawa Y, Shida D, Boku N, et al. Nutritional and inflammatory measures predict survival of patients with stage IV colorectal cancer. BMC Cancer 2020;20:1092.

13. Matsukawa T, Suto K, Kanaya M, et al. Validation and comparison of prognostic values of GNRI, PNI, and CONUT in newly diagnosed diffuse large B cell lymphoma. Ann Hematol 2020;99:2859-68.

14. Toyokawa T, Kubo N, Tamura T, et al. The pretreatment controlling nutritional status (CONUT) score is an independent prognostic factor in patients with resectable thoracic esophageal squamous cell carcinoma: results from a retrospective study. BMC Cancer 2016;16:722.

15. Terasaki F, Sugiura T, Okamura Y, et al. The preoperative controlling nutritional status (CONUT) score is an independent prognostic marker for pancreatic ductal adenocarcinoma. Updates Surg 2021;73:251-9.

16. Zhang G, Zhang Y, He F, et al. Preoperative controlling nutritional status (CONUT) score is a prognostic factor for early-stage cervical cancer patients with high-risk factors. Gynecol Oncol 2021;162:763-9.

17. Han D, Li L, Ge X, et al. MicroRNA expression integrated analysis and identification of novel biomarkers in small cell lung cancer: a meta-analysis. Transl Cancer Res 2020;9:3339-53.

18. Wang Z, Wang Y, Zhang X, et al. Pretreatment prognostic nutritional index as a prognostic factor in lung cancer: Review and meta-analysis. Clin Chim Acta 2018;486:303-10.

19. Jin S, Cao S, Xu S, et al. Clinical impact of pretreatment prognostic nutritional index (PNI) in small cell lung cancer patients treated with platinum-based chemotherapy. Clin Respir J 2018;12:2433-40.

20. Toyokawa G, Kozuma Y, Matsubara T, et al. Prognostic impact of controlling nutritional status score in resected lung squamous cell carcinoma. J Thorac Dis 2017;9:2942-51.

21. Soh J, Suzawa K, Shien K, et al. Prognostic nutrition index affects the prognosis of patients undergoing trimodality therapy for locally advanced non-small cell lung cancer. Surg Today 2020;50:1610-8.

22. Qiu C, Qu X, Shen H, et al. Evaluation of prognostic nutritional index in patients undergoing radical surgery with nonsmall cell lung cancer. Nutr Cancer 
2015;67:741-7.

23. Liu N, Jiang A, Zheng X, et al. Prognostic nutritional index identifies risk of early progression and survival outcomes in advanced non-small cell lung cancer patients treated with PD-1 inhibitors. J Cancer 2021;12:2960-7.

(English Language Editor: C. Betlzar)

Cite this article as: $\mathrm{Li} \mathrm{L}$, Wang Y, Yang $\mathrm{P}$, Xu L, Liu S, Zhang S, Weng X. Correlation of the controlling nutritional status score and the prognostic nutritional index with the prognosis of patients treated with radiotherapy for smallcell lung cancer. Ann Palliat Med 2021;10(11):11635-11642. doi: 10.21037/apm-21-2740 